\title{
SET-VALUED PERTURBATION \\ FOR TIME DEPENDENT SUBDIFFERENTIAL OPERATOR
}

\author{
Soumia SaÏdi - Mustapha Fateh Yarou
}

\begin{abstract}
In a separable Hilbert space, we consider an evolution inclusion involving time-dependent subdifferential of a proper convex lower semicontinuous function with a set-valued perturbation depending on both time and state variable. We prove, under a compactness condition on the perturbation, that there exists at least one absolutely continuous solution.
\end{abstract}

\section{Introduction}

The present work deals with perturbations of evolution equations governed by time dependent subdifferential operator of the form

$$
\left(\mathcal{P}_{F(\cdot, \cdot)}\right) \quad\left\{\begin{array}{c}
-\dot{x}(t) \in \partial \varphi(t, x(t))+F(t, x(t)) \text { for a.e. } t \in I:=\left[T_{0}, T\right], \\
x\left(T_{0}\right)=x_{0} \in \operatorname{dom} \varphi\left(T_{0}, \cdot\right),
\end{array}\right.
$$

where for each $t \in I$, the (set-valued) operator $\partial \varphi(t, \cdot)$ is the subdifferential of a time-dependent proper lower semicontinuous (lsc) convex function $\varphi(t, \cdot)$ of a separable Hilbert space $H$ into $[0,+\infty]$ and $\operatorname{dom} \varphi(t, \cdot)$ denotes the effective domain of the function $\varphi(t, \cdot)$. The set-valued mapping $F: I \times H \rightrightarrows H$ takes nonempty convex compact values. We are interested in the existence of a solution when the perturbation $F(\cdot, \cdot)$ satisfies for some compact subset $K$ of the closed

2010 Mathematics Subject Classification. 34A60, 49J52.

Key words and phrases. Evolution problem, time dependent subdifferential operator, upper hemicontinuous set-valued perturbation, absolutely continuous solution. 
unit ball $\mathbb{B}$ of $H$ and some non-negative function $\beta(\cdot) \in L_{\mathbb{R}}^{2}(I)$, the linear growth condition

$$
F(t, x) \subset \beta(t)(1+\|x\|) K, \quad \text { for all } t \in I \text { and } x \in H .
$$

The existence and uniqueness results for the unperturbed problem

$$
\left\{\begin{array}{l}
-\dot{x}(t) \in \partial \varphi(t, x(t)) \quad \text { for a.e. } t \in I, \\
x\left(T_{0}\right)=x_{0} \in \operatorname{dom} \varphi\left(T_{0}, \cdot\right),
\end{array}\right.
$$

were established by Peralba [24], [25] with an assumption expressed in terms of the conjugate function $\varphi^{*}(t, \cdot)$ of the convex function $\varphi(t, \cdot)$, that is, there exists a Lipschitz function $k: H \rightarrow \mathbb{R}_{+}$and an absolutely continuous function $a: I \rightarrow \mathbb{R}$ with $\dot{a} \in L_{\mathbb{R}}^{2}(I)$ such that, for all $x \in H$ and $s, t \in I$,

$$
\varphi^{*}(t, x) \leq \varphi^{*}(s, x)+k(x)|a(t)-a(s)| .
$$

Other results have been obtained using hypothesis required on $\varphi$ or the Moreau envelope $\varphi_{\lambda}$, see for instance [3], [21], [22], [28], [29], [31]. There are also several works dealing with set-valued or single-valued perturbations of $(\mathcal{P})$ under, in general, some compactness assumptions concerning the sublevel sets of $\varphi(t, \cdot)$ (see, e.g. [2], [6], [9], [15], [23], [27], [30]). In the line of our previous paper with single-valued Lipschitz perturbation [26], conditions on the Moreau envelope $\varphi_{\lambda}(t, \cdot)$ or the Yosida approximation of $\partial \varphi(t, \cdot)$ which cannot be translated to the new operator generated by the perturbed problem, along with compactness assumptions on the sublevel sets of $\varphi(t, \cdot)$ are not appropriate. At the opposite, as we will see below, Peralba's assumption above on the function $\varphi^{*}$ is really suited for our study in the sense that it allows us, in the setting of Hilbert space, through some ideas of Edmond and Thibault [18], [20] (see also [11]) to prove existence of absolutely continuous solution for $\left(\mathcal{P}_{F(\cdot, \cdot)}\right)$ and to avoid any compactness assumption.

For the autonomous case, that is, when $\varphi: H \rightarrow \mathbb{R} \cup\{\infty\}$ is a proper lsc convex function independent of time, we cite Attouch and Damlamian [3], Cellina and Staicu [15] and Castaing and Marcellin [11]; all these papers consider some compactness assumptions concerning the sublevel sets of $\varphi(t, \cdot)$. This hypothesis is also used in the nonautonomous case in Benabdellah and Faik [5] (see also [8]), Benabdellah, Castaing and Salvatori [6], Otani [23] and Tolstonogov [27].

In the particular case of the so-called sweeping process, i.e., for $\varphi(t, \cdot)$ taken as the indicator function of a closed moving set $C(t)$, the fixed point technique is quite efficient, under convexity assumptions of the values of both $C(\cdot)$ and $F(\cdot, \cdot)$. This is true under the additional usual assumption on $F$ requiring separate measurability with respect to $t$ and upper semicontinuity (closed graph) with respect to $x$. Results related to similar problems with nonconvex closed moving sets $C(t)$ in the finite dimensional setting for $\left(\mathcal{P}_{F(\cdot, \cdot)}\right)$, can be found on 
one hand in Castaing and Monteiro Marques [12] when $C(t)$ is the complement of an open set of $H$ and on the other hand in Castaing, Salvadori and Thibault [13] when the closed set $C(t)$ is $r$-prox-regular. In the infinite dimensional setting, recent existence theorems have been established in [19], [20], [7] and [10] when in addition to the $r$-prox-regularity of the moving set $C(t)$, a linear growth compactness condition is assumed for the set-valued mapping $F(\cdot, \cdot)$.

The paper is organized as follows. After recalling some concepts in the second section, and useful results of [24] and [25] in the third section concerning the nonautonomous case $(\mathcal{P})$, in Section 4 , we establish the existence theorem for the considered problem $\left(\mathcal{P}_{F(\cdot, \cdot)}\right)$ for a globally upper hemicontinuous perturbation. Finally, we extend this result in section 5 , to the case when the perturbation $F$ is just measurable in $t$ and upper semicontinuous in $x$.

\section{Notation and preliminaries}

Throughout the paper $I:=\left[T_{0}, T\right]\left(0 \leq T_{0}<T<+\infty\right)$ is an interval of $\mathbb{R}$ and $H$ is a real separable Hilbert space whose inner product is denoted by $\langle\cdot, \cdot\rangle$ and the associated norm by $\|\cdot\|$.

We use the following definitions and notations. We denote by $\mathbb{B}$ the closed unit ball of $H$. On the space $\mathcal{C}_{H}(I)$ of continuous maps $x: I \rightarrow H$ we consider the norm of uniform convergence on $I$. By $L_{H}^{p}(I)$ for $p \in[1,+\infty[$ (resp. $p=+\infty)$, we denote the space of measurable maps $x: I \rightarrow H$ such that $\int_{I}\|x(t)\|^{p} d t<+\infty$ (resp. which are essentially bounded) endowed with the usual norm $\|x\|_{L_{H}^{p}(I)}=\left(\int_{I}\|x(t)\|^{p} d t\right)^{1 / p}, 1 \leq p<+\infty$ (resp. endowed with the usual essential supremum norm $\|\cdot\|)$. We recall that the topological dual of $L_{H}^{1}(I)$ is $L_{H}^{\infty}(I)$.

Let $\varphi$ be a lower semicontinuous (lsc) convex function from $H$ into $\mathbb{R} \cup\{+\infty\}$ which is proper in the sense that its effective domain $\operatorname{dom} \varphi$ defined by

$$
\operatorname{dom} \varphi:=\{x \in H: \varphi(x)<+\infty\}
$$

is nonempty and, as usual, its Fenchel conjugate is defined by

$$
\varphi^{*}(v):=\sup _{x \in H}[\langle v, x\rangle-\varphi(x)] .
$$

It is often useful to regularize $\varphi$ via its Moreau envelope

$$
\varphi_{\lambda}(x):=\inf _{y \in H}\left[\varphi(y)+\frac{1}{2 \lambda}\|x-y\|^{2}\right]
$$

for $\lambda>0$. The family $\left(\varphi_{\lambda}\right)_{\lambda}$ increases when $\lambda \downarrow 0$ to the proper lsc convex function $\varphi$ and hence it epi-converges to $\varphi$ (see e.g. [1]). This entails in particular for any family $\left(x_{\lambda}\right)_{\lambda}$ of $H$ converging to $x$ that

$$
\varphi(x) \leq \liminf _{\lambda \downarrow 0} \varphi_{\lambda}\left(x_{\lambda}\right) .
$$


The Moreau envelope function $\varphi_{\lambda}$ is also known to have a Lipschitzian continuous derivative $\nabla \varphi_{\lambda}$.

The subdifferential $\partial \varphi(x)$ of $\varphi$ at $x \in \operatorname{dom} \varphi$ is

$$
\partial \varphi(x)=\{v \in H: \varphi(y) \geq\langle v, y-x\rangle+\varphi(x) \text { for all } y \in \operatorname{dom} \varphi\}
$$

and its effective domain is $\operatorname{Dom} \partial \varphi=\{x \in H: \partial \varphi(x) \neq \emptyset\}$. It is well known that if $\varphi$ is a proper lsc convex function, then its subdifferential operator $\partial \varphi$ is a maximal monotone operator. Any maximal monotone operator $A$ satisfies the closure property, that is, if $x=\lim _{n \rightarrow \infty} x_{n}$ strongly in $H$ and $y=\lim _{n \rightarrow \infty} y_{n}$ weakly in $H$, where $x_{n} \in \operatorname{Dom} A$ and $y_{n} \in A\left(x_{n}\right)$, then, $x \in \operatorname{Dom} A$ and $y \in A(x)$. For any subset $S$ of $H, \sigma(S, \cdot)$ represents the support function of $S$, that is, for all $y \in H$,

$$
\sigma(S, y):=\sup _{x \in S}\langle y, x\rangle .
$$

A set-valued mapping $F: E \rightrightarrows H$ from a Hausdorff topological space $E$ into $H$ is said to be upper semicontinuous (usc) if, for any open subset $V \subset H$, the set $\{x \in E: F(x) \subset V\}$ is open in $E$. The set-valued mapping $F$ is said to be scalarly upper semicontinuous or upper hemicontinuous if, for any $y \in H$, the real-valued function $x \mapsto \sigma(F(x), y)$ is upper semicontinuous. We refer to [4] and [14] for details concerning convex analysis and measurable set-valued mappings. We will close this section of preliminaries by recalling the following straightforward consequence of Gronwall's lemma.

Lemma $2.1([20])$. Let $\left(x_{n}(\cdot)\right)$ be a sequence of absolutely continuous maps from $I$ to $H$. Assume that $\lim _{n} x_{n}\left(T_{0}\right)=0$ and, for any $n$,

$$
\frac{d}{d t}\left(\left\|x_{n}(t)\right\|^{2}\right) \leq \beta_{n}(t)\left\|x_{n}(t)\right\|^{2}+\alpha_{n}(t) \quad \text { for a.e. } t \in I,
$$

where $\alpha_{n}(\cdot)$ and $\beta_{n}(\cdot)$ are non negative functions in $L_{\mathbb{R}}^{1}(I)$. Assume moreover that the sequence $\left(\beta_{n}(\cdot)\right)$ is bounded in $L_{\mathbb{R}}^{1}(I)$ and $\lim _{n} \int_{T_{0}}^{T} \alpha_{n}(t) d t=0$. Then,

$$
\lim _{n}\left\|x_{n}(\cdot)\right\|_{\infty}=0 .
$$

\section{Single valued time-dependent perturbation}

This section is devoted to the study of the perturbed problem

$$
\left\{\begin{array}{l}
-\dot{x}(t) \in \partial \varphi(t, x(t))+h(t), \\
x\left(T_{0}\right)=x_{0} \in \operatorname{dom} \varphi\left(T_{0}, \cdot\right),
\end{array}\right.
$$

whose perturbation is a single-valued time-dependent map. Let us first recall a result due to Peralba [24], [25].

Theorem 3.1. Let $\varphi: I \times H \rightarrow \mathbb{R}_{+} \cup\{+\infty\}$ be such that: 
$\left(\mathrm{H}_{1}\right)$ for each $t \in I$, the function $x \mapsto \varphi(t, x)$ is proper, lower semicontinuous, and convex;

$\left(\mathrm{H}_{2}\right)$ there exist a $\rho$-Lipschitzean function $k: H \rightarrow \mathbb{R}_{+}$and an absolutely continuous function $a: I \rightarrow \mathbb{R}$, with a non-negative derivative $\dot{a} \in L_{\mathbb{R}}^{2}(I)$, such that

$$
\varphi^{*}(t, x) \leq \varphi^{*}(s, x)+k(x)|a(t)-a(s)|
$$

for every $(t, s, x) \in I \times I \times H$.

Let also $x_{0} \in \operatorname{dom} \varphi\left(T_{0}, \cdot\right)$ be fixed. Then, the differential inclusion

$$
\left\{\begin{array}{l}
-\dot{x}(t) \in \partial \varphi(t, x(t)) \quad \text { for a.e. } t \in I \\
x\left(T_{0}\right)=x_{0} \in \operatorname{dom} \varphi\left(T_{0}, \cdot\right)
\end{array}\right.
$$

has a unique absolutely continuous solution $x(\cdot)$ on I. Moreover, for all $t \in I$, $x(t) \in \operatorname{dom} \varphi(t, \cdot)$ and the function $t \mapsto \varphi(t, x(t))$ is absolutely continuous on $I$.

Let us start with the following estimate which is a consequence of Propositions 3.3 and 3.4 in [26]

Proposition 3.2.

(a) The unique absolutely continuous solution $x(\cdot)$ of $(\mathcal{P})$ satisfies

$$
\begin{aligned}
\|\dot{x}\|_{L_{H}^{2}} \leq \frac{\rho}{2}\|\dot{a}\|_{L_{\mathbb{R}}^{2}}+\left[\sqrt{T-T_{0}}\right. & k(0)\|\dot{a}\|_{L_{\mathbb{R}}^{2}} \\
& \left.+\frac{\rho^{2}}{4}\|\dot{a}\|_{L_{\mathbb{R}}^{2}}^{2}+\varphi\left(T_{0}, x_{0}\right)-\varphi(T, x(T))\right]^{1 / 2} .
\end{aligned}
$$

(b) If $h \in L_{H}^{2}(I)$ and $x_{0} \in \operatorname{dom} \varphi\left(T_{0}, \cdot\right)$, then the problem $\left(\mathcal{P}_{h}\right)$ admits a unique absolutely continuous solution $x(\cdot)$ that satisfies

$$
\int_{T_{0}}^{T}\|\dot{x}(t)\|^{2} d t \leq 2 b_{0} \int_{T_{0}}^{T} \dot{a}^{2}(t) d t+\sigma \int_{T_{0}}^{T}\|h(t)\|^{2} d t+b_{1} .
$$

with

$$
\begin{aligned}
b_{0} & =\frac{1}{2}\left(k^{2}(0)+3(\rho+1)^{2}\right), \\
\sigma & =k^{2}(0)+3(\rho+1)^{2}+4, \\
b_{1} & =2\left[\left(T-T_{0}\right)+\varphi\left(T_{0}, x\left(T_{0}\right)\right)-\varphi(T, x(T))\right] .
\end{aligned}
$$

Proof. Assertion (a) corresponds to Proposition 3.3 in [26]. Concerning assertion (b), we know by Proposition 3.4 of [26] that $\left(\mathcal{P}_{h}\right)$ has a unique solution satisfying

$$
\begin{aligned}
\|\dot{x}\|_{L_{H}^{2}} \leq \frac{1}{2}(\rho+1) \| \dot{a} & +|h|\left\|_{L_{\mathbb{R}}^{2}}+\right\| h \|_{L_{H}^{2}}+\left[\sqrt{T-T_{0}} k(0)\|\dot{a}+|h|\|_{L_{\mathbb{R}}^{2}}\right. \\
+ & \left.\frac{(\rho+1)^{2}}{4}\|\dot{a}+|h|\|_{L_{\mathbb{R}}^{2}}^{2}+\varphi\left(T_{0}, x_{0}\right)-\varphi(T, x(T))\right]^{1 / 2}
\end{aligned}
$$


where $|h|$ is the function of $I$ into $\mathbb{R}$ defined by $|h|(t):=\|h(t)\|$ for all $t \in I$. Hence, observing that

$$
\begin{aligned}
\sqrt{T-T_{0}} k(0)\|\dot{a}+|h|\|_{L_{\mathbb{R}}^{2}(I)} & =2 \sqrt{T-T_{0}}\left(\frac{k(0)}{2}\|\dot{a}+|h|\|_{L_{\mathbb{R}}^{2}(I)}\right) \\
& \leq\left(T-T_{0}\right)+\frac{k^{2}(0)}{4}\|\dot{a}+|h|\|_{L_{\mathbb{R}}^{2}(I)}^{2},
\end{aligned}
$$

we obtain

$$
\begin{aligned}
& \|\dot{x}\|_{L_{H}^{2}(I)} \leq \frac{(\rho+1)}{2}\|\dot{a}+|h|\|_{L_{\mathbb{R}}^{2}(I)}+\|h\|_{L_{H}^{2}(I)} \\
+ & {\left[\left(T-T_{0}\right)+\frac{\left(k^{2}(0)+(\rho+1)^{2}\right)}{4}\|\dot{a}+|h|\|_{L_{\mathbb{R}}^{2}(I)}^{2}+\varphi\left(T_{0}, x\left(T_{0}\right)\right)-\varphi(T, x(T))\right]^{1 / 2} }
\end{aligned}
$$

and hence

$$
\begin{aligned}
& \|\dot{x}\|_{L_{H}^{2}(I)}^{2} \leq 2\left[\frac{(\rho+1)}{2}\|\dot{a}+|h|\|_{L_{\mathbb{R}}^{2}(I)}+\|h\|_{L_{H}^{2}(I)}\right]^{2} \\
+ & 2\left[\left(T-T_{0}\right)+\frac{\left(k^{2}(0)+(\rho+1)^{2}\right)}{4}\|\dot{a}+\mid h\|_{L_{\mathbb{R}}^{2}(I)}^{2}+\varphi\left(T_{0}, x\left(T_{0}\right)\right)-\varphi(T, x(T))\right] .
\end{aligned}
$$

We may also write

$$
\begin{aligned}
& \|\dot{x}\|_{L_{H}^{2}(I)}^{2} \leq(\rho+1)^{2}\|\dot{a}+|h|\|_{L_{\mathbb{R}}^{2}(I)}^{2}+4\|h\|_{L_{H}^{2}(I)}^{2} \\
+ & 2\left[\left(T-T_{0}\right)+\varphi\left(T_{0}, x\left(T_{0}\right)\right)-\varphi(T, x(T))\right]+\frac{\left(k^{2}(0)+(\rho+1)^{2}\right)}{2}\|\dot{a}+|h|\|_{L_{\mathbb{R}}^{2}(I)}^{2} .
\end{aligned}
$$

Setting

$$
b_{0}=\frac{1}{2}\left(k^{2}(0)+3(\rho+1)^{2}\right), \quad b_{1}=2\left[\left(T-T_{0}\right)+\varphi\left(T_{0}, x\left(T_{0}\right)\right)-\varphi(T, x(T))\right],
$$

one has

$$
\|\dot{x}\|_{L_{H}^{2}(I)}^{2} \leq b_{0}\|\dot{a}+|h|\|_{L_{\mathbb{R}}^{2}(I)}^{2}+4\|h\|_{L_{H}^{2}(I)}^{2}+b_{1} .
$$

As $\|\dot{a}+|h|\|_{L_{\mathbb{R}}^{2}(I)}^{2} \leq 2\|\dot{a}\|_{L_{\mathbb{R}}^{2}(I)}^{2}+2\|h\|_{L_{H}^{2}(I)}^{2}$, putting $\sigma=2\left(b_{0}+2\right)$, we get

$$
\|\dot{x}\|_{L_{H}^{2}(I)}^{2} \leq 2 b_{0}\|\dot{a}\|_{L_{\mathbb{R}}^{2}(I)}^{2}+\sigma\|h\|_{L_{H}^{2}(I)}^{2}+b_{1} .
$$

Equivalently,

$$
\int_{T_{0}}^{T}\|\dot{x}(t)\|^{2} d t \leq 2 b_{0} \int_{T_{0}}^{T} \dot{a}^{2}(t) d t+\sigma \int_{T_{0}}^{T}\|h(t)\|^{2} d t+b_{1} .
$$

\section{Set-valued perturbation}

We study here the perturbed problem $\left(\mathcal{P}_{F(\cdot, \cdot)}\right)$ under an upper hemicontinuity property for the set-valued perturbation $F$. In the development, we will use some ideas from [11], [19], [20]. 
TheOREM 4.1. Let $H$ be a real separable Hilbert space. Assume that $\varphi: I \times$ $H \rightarrow \mathbb{R}_{+} \cup\{+\infty\}$ is an extended-real-valued function satisfying $\left(\mathrm{H}_{1}\right)$ and $\left(\mathrm{H}_{2}\right)$ of Theorem 3.1. Let $F: I \times H \rightrightarrows H$ be a set-valued mapping with nonempty convex compact values such that:

(a) $F(\cdot, \cdot)$ is globally scalarly upper semicontinuous on $I \times H$;

(b) for some compact subset $K \subset \mathbb{B}$ and some non-negative function $\beta(\cdot) \in$ $L_{\mathbb{R}}^{2}(I)$, for all $(t, x) \in I \times H$, one has the growth type condition

$$
F(t, x) \subset \beta(t)(1+\|x\|) K .
$$

Then, for any $x_{0} \in \operatorname{dom} \varphi\left(T_{0}, \cdot\right)$ the following problem

$$
\left\{\begin{array}{l}
-\dot{x}(t) \in \partial \varphi(t, x(t))+F(t, x(t)) \quad \text { for a.e. } t \in I, \\
x\left(T_{0}\right)=x_{0}
\end{array}\right.
$$

has at least one absolutely continuous solution. More precisely, there exists an absolutely continuous map $x(\cdot): I \rightarrow H$ and an integrable map $y(\cdot): I \rightarrow H$ such that $x\left(T_{0}\right)=x_{0}, x(t) \in \operatorname{dom} \varphi(t, x(t))$ for all $t \in I$ and, for almost all $t \in I, y(t) \in F(t, x(t))$ and $-\dot{x}(t)-y(t) \in \partial \varphi(t, x(t))$, with

$$
\|y(t)\| \leq(\beta(t)+1)(1+\|x(t)\|) .
$$

Moreover, the following inequalities hold true

$$
\int_{T_{0}}^{T}\|\dot{x}(t)\|^{2} d t \leq \alpha+\sigma \int_{T_{0}}^{T}\|y(t)\|^{2} d t
$$

and

$$
\int_{T_{0}}^{T}\|\dot{x}(t)\|^{2} d t \leq \alpha+\sigma \int_{T_{0}}^{T}(\beta(s)+1)^{2}(1+\|x(s)\|)^{2} d s
$$

where

(4.3) $\alpha=\left(k^{2}(0)+3(\rho+1)^{2}\right) \int_{T_{0}}^{T} \dot{a}^{2}(t) d t+2\left[T-T_{0}+\varphi\left(T_{0}, x_{0}\right)-\varphi(T, x(T))\right]$,

$$
\sigma=k^{2}(0)+3(\rho+1)^{2}+4
$$

Proof. We suppose, without loss of generality, that $K$ is convex and contains 0 . If not so, we may replace $K$ by $\overline{\mathrm{co}}(K \cup\{0\})$ which is compact according to Dunford and Schwartz ([17, Theorem V.2.6]). Since the function $(1+\beta(\cdot))^{2}$ is $\lambda$-integrable on $I=\left[T_{0}, T\right]$, for the real number

$$
m=\frac{1}{4\left(T-T_{0}\right)\left(k^{2}(0)+3(\rho+1)^{2}+4\right)}>0
$$

there exists a finite subdivision $T_{0}<T_{1}<\ldots<T_{k}=T$ such that for each $j=1, \ldots, k$ one has

$$
\int_{T_{j-1}}^{T_{j}}(\beta(s)+1)^{2} d s<m
$$


Let us start first by establishing a solution on the interval $I_{1}:=\left[T_{0}, T_{1}\right]$ by constructing a sequence of maps $\left(x_{n}(\cdot)\right)$ in $\mathcal{C}_{H}\left(I_{1}\right)$ which has a subsequence converging uniformly on $I_{1}$ to a solution of $\left(\mathcal{P}_{1}\right)$.

(A) Construction of the sequence $\left(x_{n}(\cdot)\right)$.

Define, for every $n \in \mathbb{N}$, a partition of $I_{1}:=\left[T_{0}, T_{1}\right]$ with

$$
t_{i}^{n}:=T_{0}+(i-1) \frac{T_{1}-T_{0}}{n} \quad(1 \leq i \leq n+1),
$$

and consider for $i \in\{1, \ldots, n\}, \delta_{i}^{n} \in\left[t_{i}^{n}, t_{i+1}^{n}\right]$ such that

$$
\beta\left(\delta_{i}^{n}\right) \leq \inf _{t \in\left[t_{i}^{n}, t_{i+1}^{n}[\right.} \beta(t)+1 .
$$

Then, fix any $n \in \mathbb{N}$. Put $x_{1}^{n}\left(t_{1}^{n}\right)=x_{0}$ and choose $y_{1}^{n} \in F\left(\delta_{1}^{n}, x_{0}\right)$. Then, relying on Proposition 3.2, denote by $x_{1}^{n}(\cdot):\left[t_{1}^{n}, t_{2}^{n}\right] \rightarrow H$ the absolutely continuous solution on $\left[t_{1}^{n}, t_{2}^{n}\right]$ of the inclusion

$$
\left\{\begin{array}{c}
-\dot{x}(t) \in \partial \varphi(t, x(t))+y_{1}^{n} \quad \text { for a.e. } t \in\left[t_{1}^{n}, t_{2}^{n}\right], \\
x\left(t_{1}^{n}\right)=x_{1}^{n}\left(t_{1}^{n}\right)=x_{0} \in \operatorname{dom} \varphi\left(t_{1}^{n}, \cdot\right) .
\end{array}\right.
$$

Next, for each $i \in\{2, \ldots, n\}$, choose $y_{i}^{n} \in F\left(\delta_{i}^{n}, x_{i-1}^{n}\left(t_{i}^{n}\right)\right)$, and let

$$
x_{i}^{n}(\cdot):\left[t_{i}^{n}, t_{i+1}^{n}\right] \rightarrow H
$$

be the absolutely continuous solution of

$$
\left\{\begin{array}{l}
-\dot{x}(t) \in \partial \varphi(t, x(t))+y_{i}^{n} \quad \text { for a.e. } t \in\left[t_{i}^{n}, t_{i+1}^{n}\right], \\
x\left(t_{i}^{n}\right)=x_{i-1}^{n}\left(t_{i}^{n}\right) \in \operatorname{dom} \varphi\left(t_{i}^{n}, \cdot\right) .
\end{array}\right.
$$

Recall that, in view of Proposition 3.2, inequality (3.3) holds true in each subinterval $\left[t_{i}^{n}, t_{i+1}^{n}\right]$ of $I_{1}$, that is, for any $i \in\{1, \ldots, n\}$, one has

$$
\int_{t_{i}^{n}}^{t_{i+1}^{n}}\left\|\dot{x}_{i}^{n}(t)\right\|^{2} d t \leq 2 b_{0} \int_{t_{i}^{n}}^{t_{i+1}^{n}} \dot{a}^{2}(t) d t+\sigma \int_{t_{i}^{n}}^{t_{i+1}^{n}}\left\|y_{i}^{n}\right\|^{2} d t+c_{i} .
$$

with

$$
\begin{aligned}
b_{0} & =\frac{1}{2}\left(k^{2}(0)+3(\rho+1)^{2}\right), \\
\sigma & =k^{2}(0)+3(\rho+1)^{2}+4, \\
c_{i} & =2\left[\left(t_{i+1}^{n}-t_{i}^{n}\right)+\varphi\left(t_{i}^{n}, x_{i}^{n}\left(t_{i}^{n}\right)\right)-\varphi\left(t_{i+1}^{n}, x_{i}^{n}\left(t_{i+1}^{n}\right)\right)\right] .
\end{aligned}
$$

Now, define $x_{n}:\left[T_{0}, T_{1}\right] \rightarrow H$ by

$$
x_{n}(t)= \begin{cases}x_{i}^{n}(t) & \text { if } t \in\left[t_{i}^{n}, t_{i+1}^{n}[\text { for some } i \in\{1, \ldots, n\},\right. \\ x_{n}^{n}\left(T_{1}\right) & \text { if } t=T_{1} .\end{cases}
$$


Such a map $x_{n}(\cdot)$ is absolutely continuous on $\left[T_{0}, T_{1}\right]$. Consider the maps $\theta_{n}, \Delta_{n}:\left[T_{0}, T_{1}\right] \rightarrow\left[T_{0}, T_{1}\right]$ such that

$$
\theta_{n}(t)= \begin{cases}t_{i}^{n} & \text { if } t \in\left[t_{i}^{n}, t_{i+1}^{n}[\text { for some } i \in\{1, \ldots, n\}\right. \\ T_{1} & \text { if } t=T_{1}\end{cases}
$$

and

$$
\Delta_{n}(t)= \begin{cases}\delta_{i}^{n} & \text { if } t \in\left[t_{i}^{n}, t_{i+1}^{n}[\text { for some } i \in\{1, \ldots, n\},\right. \\ \delta_{n}^{n} & \text { if } t=T_{1}\end{cases}
$$

Next, define $y_{n}:\left[T_{0}, T_{1}\right] \rightarrow H$ by

$$
y_{n}(t)= \begin{cases}y_{i}^{n} & \text { if } t \in\left[t_{i}^{n}, t_{i+1}^{n}[\text { for some } i \in\{1, \ldots, n\},\right. \\ y_{n}^{n} & \text { if } t=T_{1} .\end{cases}
$$

Then, for each $n \in \mathbb{N}$, we have the following:

(1) $y_{n}(t) \in F\left(\Delta_{n}(t), x_{n}\left(\theta_{n}(t)\right)\right) \subset \beta\left(\Delta_{n}(t)\right)\left(1+\left\|x_{n}\left(\theta_{n}(t)\right)\right\|\right) K$, for all $t \in$ $\left[T_{0}, T_{1}\right]$

(2) for all $t \in\left[T_{0}, T_{1}\right],\left\|y_{n}(t)\right\| \leq \beta\left(\Delta_{n}(t)\right)\left(1+\left\|x_{n}\left(\theta_{n}(t)\right)\right\|\right)$,

(3) $x_{n}\left(T_{0}\right)=x_{0}$

(4) $-\dot{x}_{n}(t) \in \partial \varphi\left(t, x_{n}(t)\right)+y_{n}(t)$ for almost every $t \in\left[T_{0}, T_{1}\right]$, and hence $-\dot{x}_{n}(t) \in \partial \varphi\left(t, x_{n}(t)\right)+F\left(\Delta_{n}(t), x_{n}\left(\theta_{n}(t)\right)\right)$ for a.e. $t \in\left[T_{0}, T_{1}\right]$.

Further, we may write (4.8), as follows

$$
\int_{t_{i}^{n}}^{t_{i+1}^{n}}\left\|\dot{x}_{n}(t)\right\|^{2} d t \leq 2 b_{0} \int_{t_{i}^{n}}^{t_{i+1}^{n}} \dot{a}^{2}(t) d t+\sigma \int_{t_{i}^{n}}^{t_{i+1}^{n}}\left\|y_{n}(t)\right\|^{2} d t+c_{i} .
$$

Taking (2) and (4.7) into account, it results that, for any $i \in\{1, \ldots, n\}$,

$$
\begin{aligned}
\int_{t_{i}^{n}}^{t_{i+1}^{n}} & \left\|\dot{x}_{n}(t)\right\|^{2} d t \\
& \leq 2 b_{0} \int_{t_{i}^{n}}^{t_{i+1}^{n}} \dot{a}^{2}(t) d t+\sigma \int_{t_{i}^{n}}^{t_{i+1}^{n}}(\beta(t)+1)^{2}\left(1+\left\|x_{n}\left(\theta_{n}(t)\right)\right\|\right)^{2} d t+c_{i} \\
& \leq 2 b_{0} \int_{t_{i}^{n}}^{t_{i+1}^{n}} \dot{a}^{2}(t) d t+\sigma\left(1+\left\|x_{n}\left(t_{i}^{n}\right)\right\|\right)^{2} \int_{t_{i}^{n}}^{t_{i+1}^{n}}(\beta(t)+1)^{2} d t+c_{i} \\
& \leq 2 b_{0} \int_{t_{i}^{n}}^{t_{i+1}^{n}} \dot{a}^{2}(t) d t+\sigma\left(1+\max _{1 \leq i \leq n+1}\left\|x_{n}\left(t_{i}^{n}\right)\right\|\right)^{2} \int_{t_{i}^{n}}^{t_{i+1}^{n}}(\beta(t)+1)^{2} d t+c_{i},
\end{aligned}
$$

and, with this being true for any $i \in\{1, \ldots, n\}$, we obtain

$$
\sum_{i=1}^{n} \int_{t_{i}^{n}}^{t_{i+1}^{n}}\left\|\dot{x}_{n}(t)\right\|^{2} d t \leq 2 b_{0} \int_{T_{0}}^{T_{1}} \dot{a}^{2}(t) d t+\sigma\left(1+\left\|x_{n}(\cdot)\right\|_{\infty}\right)^{2} \int_{T_{0}}^{T_{1}}(\beta(t)+1)^{2} d t+c_{n}^{\prime}
$$


where $\|\cdot\|_{\infty}$ denotes the supremum norm over the interval $\left[T_{0}, T_{1}\right]$ and

$$
c_{n}^{\prime}=\sum_{i=1}^{n} c_{i}=2\left[T_{1}-T_{0}+\varphi\left(T_{0}, x_{0}\right)-\varphi\left(T_{1}, x_{n}\left(T_{1}\right)\right)\right] .
$$

As $-\varphi\left(T_{1}, x_{n}\left(T_{1}\right)\right) \leq 0$, putting $d=2\left[T_{1}-T_{0}+\varphi\left(T_{0}, x_{0}\right)\right]$, we may write

$$
\int_{T_{0}}^{T_{1}}\left\|\dot{x}_{n}(t)\right\|^{2} d t \leq 2 b_{0} \int_{T_{0}}^{T_{1}} \dot{a}^{2}(t) d t+2 \sigma\left(1+\left\|x_{n}(\cdot)\right\|_{\infty}^{2}\right) \int_{T_{0}}^{T_{1}}(\beta(t)+1)^{2} d t+d
$$

and hence

$$
\int_{T_{0}}^{T_{1}}\left\|\dot{x}_{n}(t)\right\|^{2} d t \leq b+c\left\|x_{n}(\cdot)\right\|_{\infty}^{2}
$$

where

$b=2 b_{0} \int_{T_{0}}^{T_{1}} \dot{a}^{2}(t) d t+2 \sigma \int_{T_{0}}^{T_{1}}(\beta(t)+1)^{2} d t+d \quad$ and $\quad c=2 \sigma \int_{T_{0}}^{T_{1}}(\beta(t)+1)^{2} d t$.

Using the Cauchy-Schwartz inequality and (4.10), one has for all $s \in I_{1}$

$$
\left\|x_{n}(s)-x_{0}\right\|^{2} \leq\left(s-T_{0}\right)\left(\int_{T_{0}}^{s}\left\|\dot{x}_{n}(t)\right\|^{2} d t\right) \leq\left(T_{1}-T_{0}\right)\left(b+c\left\|x_{n}(\cdot)\right\|_{\infty}^{2}\right)
$$

and hence

$$
\left\|x_{n}(s)\right\|^{2} \leq 2\left\|x_{0}\right\|^{2}+2\left\|x_{n}(s)-x_{0}\right\|^{2} \leq 2\left\|x_{0}\right\|^{2}+2\left(T_{1}-T_{0}\right)\left(b+c\left\|x_{n}(\cdot)\right\|_{\infty}^{2}\right) .
$$

Consequently, for each $n$, we get

$$
\left(1-2\left(T_{1}-T_{0}\right) c\right)\left\|x_{n}(\cdot)\right\|_{\infty}^{2} \leq 2\left(\left\|x_{0}\right\|^{2}+\left(T-T_{0}\right) b\right) .
$$

According to (4.6), that is, $2\left(T_{1}-T_{0}\right) c<1$, one has, for any $t$ and for any $n$,

$$
\left\|x_{n}(\cdot)\right\|_{\infty} \leq M_{1}
$$

where

$$
M_{1}:=\left(\frac{2\left(\left\|x_{0}\right\|^{2}+\left(T_{1}-T_{0}\right) b\right)}{1-2\left(T_{1}-T_{0}\right) c}\right)^{1 / 2} .
$$

For each $n \in \mathbb{N}$ and any $t \in I_{1}:=\left[T_{0}, T_{1}\right]$, define $z_{n}(t):=\int_{T_{0}}^{t} y_{n}(s) d s$. Then, the map $z_{n}(\cdot)$ is absolutely continuous on $\left[T_{0}, T_{1}\right]$. By virtue of (2), (4.7) and (4.11), for $T_{0} \leq r \leq t \leq T_{1}$, we have

$$
\left\|y_{n}(t)\right\| \leq\left(M_{1}+1\right)(\beta(t)+1)
$$

and

$$
\left\|z_{n}(t)-z_{n}(r)\right\| \leq\left(M_{1}+1\right) \int_{r}^{t}(\beta(s)+1) d s
$$

so that the family $\left(z_{n}\right)_{n \in \mathbb{N}}$ is equicontinuous in $\mathcal{C}_{H}\left(I_{1}\right)$.

Furthermore, since $K$ is convex with $0 \in K$, it follows from (1), (4.7) and (4.11) that

$$
\forall n \in \mathbb{N}, \forall t \in\left[T_{0}, T_{1}\right], \quad y_{n}(t) \in\left(M_{1}+1\right)(\beta(t)+1) K .
$$


Since $K$ is closed and convex, this yields that for all $n \geq 1$ and $t \in\left[T_{0}, T_{1}\right]$

$$
z_{n}(t) \in\left[\left(M_{1}+1\right) \int_{T_{0}}^{t}(\beta(s)+1) d s\right] K
$$

and once more, since $K$ is convex with $0 \in K$, we deduce that for any $t \in\left[T_{0}, T_{1}\right]$ the set $\left\{z_{n}(t), n \in \mathbb{N}\right\}$ is included in the strongly compact set

$$
\left[\left(M_{1}+1\right) \int_{T_{0}}^{T_{1}}(\beta(s)+1) d s\right] K .
$$

(B) Uniform convergence of a subsequence of $\left(x_{n}(\cdot)\right)$ to some map $u_{1}(\cdot)$.

Ascoli's theorem ensures us that, up to a subsequence, $\left(z_{n}\right)$ converges uniformly on $\left[T_{0}, T_{1}\right]$ to some continuous mapping $z(\cdot)$. Further, (4.10) and (4.11) entail that

$$
\sup _{n \in \mathbb{N}}\left\|\dot{x}_{n}(\cdot)\right\|_{L_{H}^{2}\left(I_{1}\right)}<+\infty .
$$

Now, making use of the monotonicity of $\partial \varphi(t, \cdot)$ for all $t \in I_{1}$, we will show that the corresponding subsequence $\left(x_{n}\right)$ converges uniformly on $I_{1}$ to some solution over $I_{1}$ of the differential inclusion under consideration. For any $n \in \mathbb{N}$ and any $t \in\left[T_{0}, T_{1}\right]$, define $X_{n}(t):=x_{n}(t)+z_{n}(t)$. The maps $X_{n}$ are clearly absolutely continuous and for any fixed $p, q \in \mathbb{N}$, and for almost all $t \in\left[T_{0}, T_{1}\right]$, one has

$$
\begin{aligned}
\frac{1}{2} \frac{d}{d t} \| X_{p}(t) & -X_{q}(t) \|^{2}=\left\langle\dot{X}_{p}(t)-\dot{X}_{q}(t), X_{p}(t)-X_{q}(t)\right\rangle \\
& =\left\langle\dot{X}_{p}(t)-\dot{X}_{q}(t), x_{p}(t)-x_{q}(t)\right\rangle+\left\langle\dot{X}_{p}(t)-\dot{X}_{q}(t), z_{p}(t)-z_{q}(t)\right\rangle .
\end{aligned}
$$

By definition, one has

$$
\begin{aligned}
& -\dot{X}_{p}(t)=-\dot{x}_{p}(t)-y_{p}(t) \in \partial \varphi\left(t, x_{p}(t)\right), \\
& -\dot{X}_{q}(t)=-\dot{x}_{q}(t)-y_{q}(t) \in \partial \varphi\left(t, x_{q}(t)\right),
\end{aligned}
$$

and the monotonicity property of $\partial \varphi(t, \cdot)$ entails that

$$
\left\langle\dot{X}_{p}(t)-\dot{X}_{q}(t), x_{p}(t)-x_{q}(t)\right\rangle \leq 0 .
$$

Therefore, one has

$$
\frac{1}{2} \frac{d}{d t}\left\|X_{p}(t)-X_{q}(t)\right\|^{2} \leq\left\|\dot{X}_{p}(t)-\dot{X}_{q}(t)\right\|\left\|z_{p}(t)-z_{q}(t)\right\| .
$$

Now, we deduce from (4.15) that the sequence $\left(\dot{x}_{n}\right)$ is bounded in $L_{H}^{2}\left(I_{1}\right)$, and since via (4.12)

$$
\sup _{n \in \mathbb{N}}\left\|\dot{z}_{n}(\cdot)\right\|_{L_{H}^{2}\left(I_{1}\right)}^{2} \leq\left(M_{1}+1\right)^{2} \int_{T_{0}}^{T_{1}}(\beta(s)+1)^{2} d s<+\infty,
$$

we conclude that

$$
A:=\sup _{n \in \mathbb{N}}\left\|\dot{X}_{n}(\cdot)\right\|_{L_{H}^{2}\left(I_{1}\right)}<+\infty
$$


The uniform convergence of the sequence $\left(z_{n}\right)$ ensures us that

$$
\int_{T_{0}}^{T_{1}}\left\|z_{p}(t)-z_{q}(t)\right\| d t \rightarrow 0
$$

when $p, q \rightarrow \infty$. This, along with the fact that $\left\|X_{p}\left(T_{0}\right)-X_{q}\left(T_{0}\right)\right\|=0$, entails

$$
\lim _{p, q \rightarrow \infty}\left\|X_{p}(\cdot)-X_{q}(\cdot)\right\|_{\infty}=0 .
$$

Then, the uniform Cauchy's criterion guarantees that the sequence $\left(X_{n}(\cdot)\right)$ converges uniformly on $I_{1}$ to some map $X(\cdot) \in \mathcal{C}_{H}\left(I_{1}\right)$. So, the sequence $\left(x_{n}\right)=$ $\left(X_{n}-z_{n}\right)$ converges uniformly on $I_{1}$ to some continuous map $u_{1}(\cdot) \in \mathcal{C}_{H}\left(I_{1}\right)$, with $u_{1}\left(T_{0}\right)=x_{0}$ according to (3). By (4.15) the sequence $\left(\dot{x}_{n}\right)$ is bounded in $L_{H}^{\infty}\left(I_{1}\right)$ and hence also in $L_{H}^{2}\left(I_{1}\right)$. We may then extract a subsequence converging weakly in $L_{H}^{2}\left(I_{1}\right)$ to some map $v(\cdot)$. The equality

$$
x_{n}(t)=x_{n}\left(T_{0}\right)+\int_{T_{0}}^{t} \dot{x}_{n}(s) d s \quad \text { for all } t \in I_{1}
$$

then yields

$$
u_{1}(t)=u_{1}\left(T_{0}\right)+\int_{T_{0}}^{t} v(s) d s \quad \text { for all } t \in I_{1}
$$

and hence the map $u_{1}(\cdot)$ is absolutely continuous on $I_{1}$ with $\dot{u}_{1}(\cdot)=v(\cdot)$ on $I_{1}$.

(C) Let us prove that $u_{1}(\cdot)$ is a solution of $\left(\mathcal{P}_{1}\right)$ on $I_{1}$.

Recall that, for almost all $t \in\left[T_{0}, T_{1}\right]$, for all $n \in \mathbb{N}$ one has

$$
-\dot{X}_{n}(t) \in \partial \varphi\left(t, x_{n}(t)\right) \quad \text { and } \quad y_{n}(t) \in F\left(\Delta_{n}(t), x_{n}\left(\theta_{n}(t)\right)\right)
$$

where $\lim _{n \rightarrow \infty} \max \left\{\left|\triangle_{n}(t)-t\right| ;\left|\theta_{n}(t)-t\right|\right\}=0$ and that by (4.12) one also has

$$
\sup _{n \in \mathbb{N}}\left\|y_{n}(\cdot)\right\|_{L_{H}^{2}\left(I_{1}\right)}^{2} \leq\left(M_{1}+1\right)^{2} \int_{T_{0}}^{T_{1}}(\beta(s)+1)^{2} d s<+\infty .
$$

We may assume that the sequences $\left(y_{n}\right)$ and $\left(\dot{x}_{n}\right)$ converge weakly in $L_{H}^{2}\left(\left[T_{0}, T_{1}\right]\right)$ to $y^{1}$ and $\dot{u}_{1}$ respectively (see (4.16)). Then, the corresponding subsequence $\left(\dot{X}_{n}\right)$ converges weakly in $L_{H}^{2}\left(\left[T_{0}, T_{1}\right]\right)$ to $y^{1}+\dot{u}_{1}$. Classically, following the corresponding arguments of the proof of Theorem 1 in [20], one has

$$
-\dot{u}_{1}(t) \in \partial \varphi\left(t, u_{1}(t)\right)+y^{1}(t) \quad \text { for a.e. } t \in\left[T_{0}, T_{1}\right] .
$$

It remains to show that $y^{1}(t) \in F\left(t, u_{1}(t)\right)$ for almost every $t \in\left[T_{0}, T_{1}\right]$. By construction, we have $y_{n}(t) \in F\left(\Delta_{n}(t), x_{n}\left(\theta_{n}(t)\right)\right)$ for almost every $t \in\left[T_{0}, T_{1}\right]$. As $\left(\triangle_{n}(t), x_{n}\left(\theta_{n}(t)\right)\right)$ converges to $\left(t, u_{1}(t)\right)$ for each $t \in\left[T_{0}, T_{1}\right]$ and $\left(y_{n}\right)$ converges weakly in $L_{H}^{2}\left(\left[T_{0}, T_{1}\right]\right)$ to $y^{1}$, and $F$ is scalarly upper semicontinuous on $\left[T_{0}, T_{1}\right] \times H$, invoking the closure theorem in [4, Theorem 1.4.1], we get the required inclusion. Combining this with $(4.17)$, we conclude that $u_{1}(\cdot)$ is an 
absolutely continuous solution of $-\dot{u}_{1}(t) \in \partial \varphi\left(t, u_{1}(t)\right)+F\left(t, u_{1}(t)\right)$ for almost every $t \in\left[T_{0}, T_{1}\right], u_{1}\left(T_{0}\right)=x_{0}$ over $I_{1}$. Summing (4.9) it follows that

$$
\sum_{i=1}^{n} \int_{t_{i}^{n}}^{t_{i+1}^{n}}\left\|\dot{x}_{n}(t)\right\|^{2} d t \leq 2 b_{0} \sum_{i=1}^{n} \int_{t_{i}^{n}}^{t_{i+1}^{n}} \dot{a}^{2}(t) d t+\sigma \sum_{i=1}^{n} \int_{t_{i}^{n}}^{t_{i+1}^{n}}\left\|y_{n}(t)\right\|^{2} d t+\sum_{i=1}^{n} c_{i}
$$

and hence, for all $n$, we have

$$
\int_{T_{0}}^{T_{1}}\left\|\dot{x}_{n}(t)\right\|^{2} d t \leq 2 b_{0} \int_{T_{0}}^{T_{1}} \dot{a}^{2}(t) d t+\sigma \int_{T_{0}}^{T_{1}}\left\|y_{n}(t)\right\|^{2} d t+c_{n}^{\prime} .
$$

Taking (4.7) and (1) into account we obtain

$$
\begin{aligned}
\int_{T_{0}}^{T_{1}} & \left\|\dot{x}_{n}(t)\right\|^{2} d t \\
& \leq 2 b_{0} \int_{T_{0}}^{T_{1}} \dot{a}^{2}(t) d t+\sigma \int_{T_{0}}^{T_{1}}(\beta(t)+1)^{2}\left(1+\left\|x_{n}\left(\theta_{n}(t)\right)\right\|\right)^{2} d t+c_{n}^{\prime} .
\end{aligned}
$$

As an estimate on the velocity, let us underline that, taking the superior limit on $n$ in (4.18) and using the preceding convergence results yield

$$
\int_{T_{0}}^{T_{1}}\left\|\dot{u}_{1}(t)\right\|^{2} d t \leq 2 b_{0} \int_{T_{0}}^{T_{1}} \dot{a}^{2}(t) d t+\sigma \int_{T_{0}}^{T_{1}}\left\|y^{1}(t)\right\|^{2} d t+\underset{n}{\limsup c_{n}^{\prime} .}
$$

Since $x_{n}(t) \rightarrow u_{1}(t)$, by the lower semicontinuity of $\varphi(t, \cdot)$, we have

$$
\begin{aligned}
\underset{n}{\limsup } c_{n}^{\prime} & =2\left[T_{1}-T_{0}+\varphi\left(T_{0}, x_{0}\right)-\liminf _{n} \varphi\left(T_{1}, x_{n}\left(T_{1}\right)\right)\right] \\
& \leq 2\left[T_{1}-T_{0}+\varphi\left(T_{0}, x_{0}\right)-\varphi\left(T_{1}, u_{1}\left(T_{1}\right)\right)\right] .
\end{aligned}
$$

Hence, we obtain

$$
\int_{T_{0}}^{T_{1}}\left\|\dot{u}_{1}(t)\right\|^{2} d t \leq \alpha_{1}+\sigma \int_{T_{0}}^{T_{1}}\left\|y^{1}(t)\right\|^{2} d t
$$

where

$$
\alpha_{1}=\left(k^{2}(0)+3(\rho+1)^{2}\right) \int_{T_{0}}^{T_{1}} \dot{a}^{2}(t) d t+2\left[T_{1}-T_{0}+\varphi\left(T_{0}, x_{0}\right)-\varphi\left(T_{1}, u_{1}\left(T_{1}\right)\right)\right] .
$$

Similarly, taking the superior limit on $n$ in (4.19) and using the preceding convergence results again yield

$$
\int_{T_{0}}^{T_{1}}\left\|\dot{u}_{1}(t)\right\|^{2} d t \leq \alpha_{1}+\sigma \int_{T_{0}}^{T_{1}}(\beta(t)+1)^{2}\left(1+\left\|u_{1}(t)\right\|\right)^{2} d t .
$$

The analysis above also yields a solution $u_{2}(\cdot)$ to the differential inclusion $\left(\mathcal{P}_{1}\right)$ on the interval $I_{2}:=\left[T_{1}, T_{2}\right]$ with the initial condition $u_{2}\left(T_{1}\right)=u_{1}\left(T_{1}\right)$ and by (4.20) and (4.21) the solution satisfies for

$$
\alpha_{2}=\left(k^{2}(0)+3(\rho+1)^{2}\right) \int_{T_{1}}^{T_{2}} \dot{a}^{2}(t) d t+2\left[T_{2}-T_{1}+\varphi\left(T_{1}, u_{1}\left(T_{1}\right)\right)-\varphi\left(T_{2}, u_{2}\left(T_{2}\right)\right)\right]
$$


and for some $L^{2}\left(I_{2}\right)$-selection $y^{2}(\cdot)$ of $F\left(\cdot, u_{2}(\cdot)\right)$ we have the inequalities

$$
\int_{T_{1}}^{T_{2}}\left\|\dot{u}_{2}(t)\right\|^{2} d t \leq \alpha_{2}+\sigma \int_{T_{1}}^{T_{2}}\left\|y^{2}(t)\right\|^{2} d t
$$

and

$$
\int_{T_{1}}^{T_{2}}\left\|\dot{u}_{2}(t)\right\|^{2} d t \leq \alpha_{2}+\sigma \int_{T_{1}}^{T_{2}}(\beta(t)+1)^{2}\left(1+\left\|u_{2}(t)\right\|\right)^{2} d t .
$$

Proceeding in a similar way we obtain $u_{3}(\cdot)$ on $\left[T_{2}, T_{3}\right], \ldots, u_{k}(\cdot)$ on $\left[T_{k-1}, T_{k}\right]$. Putting $x(t)=u_{j}(t)$ and $y(t)=y^{j}(t)$ if $t \in\left[T_{j-1}, T_{j}\right]$, we see that $x(\cdot)$ is an absolutely continuous solution of $\left(\mathcal{P}_{1}\right)$ on the whole interval $I=\left[T_{0}, T\right]$ and the estimations (4.1) and (4.2) of the theorem hold because

$\alpha:=\sum_{j=1}^{k} \alpha_{j}=\left(k^{2}(0)+3(\rho+1)^{2}\right) \int_{T_{0}}^{T} \dot{a}^{2}(t) d t+2\left[T-T_{0}+\varphi\left(T_{0}, x_{0}\right)-\varphi(T, x(T))\right]$.

The proof of the theorem is then complete.

As a consequence, we have the following properties

Proposition 4.2. The absolutely continuous solution $x(\cdot)$ of $\left(\mathcal{P}_{1}\right)$ satisfies

$$
\int_{T_{0}}^{T}\|\dot{x}(t)\|^{2} d t \leq \alpha+\sigma(1+l)^{2} \int_{T_{0}}^{T}(\beta(t)+1)^{2} d t,
$$

and $y(t) \in(1+l)(\beta(t)+1) \overline{\mathrm{co}}(K \cup\{0\}),\|y(t)\| \leq(\beta(t)+1)(1+l)$ for almost every $t \in I$ with $l:=\left\|x_{0}\right\|+[\xi(T)]^{1 / 2}$ and where $\xi(\cdot)$ is the increasing, continuous, and non-negative function defined on $\left[T_{0}, T\right]$ by

$$
\xi(s)=b(s)+2 \sigma\left(s-T_{0}\right) \int_{T_{0}}^{s} b(\tau)(\beta(\tau)+1)^{2} \exp \left(2 \sigma \int_{\tau}^{s} \theta(\beta(\theta)+1)^{2} d \theta\right) d \tau,
$$

and, for each $t \in\left[T_{0}, T\right]$,

$$
b(t)=\left(t-T_{0}\right)\left[\alpha+2 \sigma\left(1+\left\|x_{0}\right\|\right)^{2} \int_{T_{0}}^{t}(\beta(\tau)+1)^{2} d \tau\right] .
$$

The constants $\alpha$ and $\sigma$ are defined as in Theorem 4.1.

Proof. Owing to (4.2) and making use of the absolute continuity of $x(\cdot)$ on $\left[T_{0}, T\right]$, we may write, for $T_{0} \leq s<T$,

$$
\begin{aligned}
\left\|x(s)-x_{0}\right\|^{2} & \leq\left(s-T_{0}\right) \int_{T_{0}}^{s}\|\dot{x}(\tau)\|^{2} d \tau \\
& \leq\left(s-T_{0}\right)\left[\alpha+\sigma \int_{T_{0}}^{s}(\beta(\tau)+1)^{2}(1+\|x(\tau)\|)^{2} d \tau\right] .
\end{aligned}
$$


Hence, for any $s \in\left[T_{0}, T\right]$,

$$
\begin{aligned}
\left\|x(s)-x_{0}\right\|^{2} \leq\left(s-T_{0}\right)[\alpha+2 \sigma(1+ & \left.\left\|x_{0}\right\|\right)^{2} \int_{T_{0}}^{s}(\beta(\tau)+1)^{2} d \tau \\
& \left.+2 \sigma \int_{T_{0}}^{s}(\beta(\tau)+1)^{2}\left\|x(\tau)-x_{0}\right\|^{2} d \tau\right] .
\end{aligned}
$$

Applying Gronwall's inequality entails that given $s \in\left[T_{0}, T\right]$, one has

$$
\left\|x(s)-x_{0}\right\|^{2} \leq \xi(s),
$$

where

$$
\xi(s)=b(s)+c(s) \int_{T_{0}}^{s} b(\tau)(\beta(\tau)+1)^{2} \exp \left(\int_{\tau}^{s}(\beta(\theta)+1)^{2} c(\theta) d \theta\right) d \tau
$$

with

$$
\begin{aligned}
& b(t)=\left(t-T_{0}\right)\left[\alpha+2 \sigma\left(1+\left\|x_{0}\right\|\right)^{2} \int_{T_{0}}^{t}(\beta(\tau)+1)^{2} d \tau\right], \\
& c(t)=2 \sigma\left(t-T_{0}\right) .
\end{aligned}
$$

Clearly such functions $b(\cdot), c(\cdot)$ and $\xi(\cdot)$ are increasing and continuous on $\left[T_{0}, T\right]$. Indeed, as a straight consequence of (4.22) and the finiteness of $T$, one has $\|x(\cdot)\|_{\infty} \leq l$, where $l:=\left\|x_{0}\right\|+[\xi(T)]^{1 / 2}$. Consequently,

$$
\|y(t)\| \leq(\beta(t)+1)(1+l) \quad \text { for a.e. } t \in I .
$$

\section{Separately scalarly u.s.c. perturbation}

In this section we weaken the assumption of Theorem 4.1 concerning the setvalued map $F$. Here, it is assumed to be separately scalarly upper semicontinuous on $H$ and to have measurable selection with respect to the first variable. The development is for a large an adaptation of [19], [20]. In the remaining of the paper, we will denote by $\alpha, \sigma$, and $m$ the constants defined in Section 4 , by (4.3), (4.4) and (4.5), respectively.

To begin with, we suppose that the function $\beta(\cdot)$ in the growth condition is constant.

Theorem 5.1. Under assumptions of Theorem 4.1 on $\varphi$, let $F: I \times H \rightrightarrows H$ be a set-valued mapping with nonempty convex compact values such that

(a) for any $x \in H, F(\cdot, x)$ has a $\lambda$-measurable selection;

(b) for all $t \in I, F(t, \cdot)$ is scalarly upper semicontinuous on $H$;

(c) for some compact subset $K \subset \mathbb{B}$ and some real number $\beta>0$, for all $(t, x) \in I \times H$, one has

$$
F(t, x) \subset \beta(1+\|x\|) K .
$$


Then, for any $x_{0} \in \operatorname{dom} \varphi\left(T_{0}, \cdot\right)$ the following problem

$$
\left\{\begin{array}{l}
-\dot{x}(t) \in \partial \varphi(t, x(t))+F(t, x(t)) \quad \text { for a.e. } t \in I, \\
x\left(T_{0}\right)=x_{0},
\end{array}\right.
$$

has at least one absolutely continuous solution. More precisely, there exist an absolutely continuous map $x(\cdot): I \rightarrow H$ and an integrable map $z(\cdot): I \rightarrow H$ such that $x\left(T_{0}\right)=x_{0}, x(t) \in \operatorname{dom} \varphi(t, x(t))$ for all $t \in I$ and for almost all $t \in I$, $z(t) \in F(t, x(t))$ and $-\dot{x}(t)-z(t) \in \partial \varphi(t, x(t))$ and

$$
z(t) \in(\beta+1)(1+\|x(t)\|) \overline{\mathrm{co}}(K \cup\{0\}) .
$$

Moreover, the following inequalities hold true

$$
\int_{T_{0}}^{T}\|\dot{x}(t)\|^{2} d t \leq \alpha+\sigma \int_{T_{0}}^{T}\|z(t)\|^{2} d t
$$

and

$$
\int_{T_{0}}^{T}\|\dot{x}(t)\|^{2} d t \leq \alpha+\sigma(\beta+1)^{2} \int_{T_{0}}^{T}(1+\|x(t)\|)^{2} d t .
$$

Proof. We will reduce the problem to the previous case, via set-valued versions of Scorza-Dragoni's theorem and Dugundji's extension theorem, and construct a sequence of absolutely continuous maps $\left(x_{n}(\cdot)\right)$. Next, it will be proved that this sequence has a subsequence converging uniformly in $\mathcal{C}_{H}(I)$ to a solution of $\left(\mathcal{P}_{2}\right)$.

We suppose without loss of generality, that $K$ is convex and contains 0 . If not so, we may replace $K$ by $\overline{c o}(K \cup\{0\})$. Dividing, if necessary $I$ into intervals of a same suitable length, we may suppose also that,

$$
(\beta+1)^{2}\left(T-T_{0}\right)<m .
$$

(A) Existence of the sequence $\left(x_{n}(\cdot)\right)$.

Set for the real number

$$
\begin{gathered}
\alpha_{0}=\left(k^{2}(0)+3(\rho+1)^{2}\right) \int_{T_{0}}^{T} \dot{a}^{2}(t) d t+2\left[T-T_{0}+\varphi\left(T_{0}, x_{0}\right)\right], \\
M_{2}:=\left(\frac{2\left(\left\|x_{0}\right\|^{2}+\left(T-T_{0}\right)\left[\alpha_{0}+2 \sigma(\beta+1)^{2}\left(T-T_{0}\right)\right]\right)}{1-4\left(T-T_{0}\right)^{2} \sigma(\beta+1)^{2}}\right)^{1 / 2},
\end{gathered}
$$

and fix a continuous function $\phi: \mathbb{R}^{+} \rightarrow[0,1]$ such that

$$
\phi(\tau)= \begin{cases}1 & \text { if } \tau \leq M_{2} \\ 0 & \text { if } \tau \geq M_{2}+1\end{cases}
$$

Let us consider the compact convex metric space $Y:=\beta\left(2+M_{2}\right) K$, which is a Borel subset of $H$, and let us define a set-valued map $\widehat{F}: I \times H \rightrightarrows Y$ by

$$
\widehat{F}(t, x):=\phi(\|x\|) F(t, x) .
$$


Obviously, $\widehat{F}(\cdot, x)$ has a measurable selection for all $x \in H$ and, for each $t \in\left[T_{0}, T\right]$, the graph of $\widehat{F}(t, \cdot)$ is closed in $H \times Y$. Therefore, according to the set-valued version of Scorza-Dragoni's theorem from Castaing and Monteiro Marques [12], there exists a set-valued map $\widetilde{F}: I \times H \rightrightarrows Y$ with convex compact (possibly empty) values such that:

- for some $\lambda$-negligible subset $N_{0} \subset I$, for all $t \in I \backslash N_{0}$ and for all $x \in H$,

$$
\widetilde{F}(t, x) \subset \widehat{F}(t, x) \text {; }
$$

- there exists an increasing sequence $\left(I_{n}\right)_{n \geq 1}$ of compact subsets of $I$ such that, for each $n \geq 1, \lambda\left(I \backslash I_{n}\right) \leq 1 / n$ and the restriction of $\widetilde{F}$ to $I_{n} \times H$, denoted by $\left.\widetilde{F}\right|_{I_{n} \times H}$, is (globally) upper semicontinuous with nonempty convex compact values.

By the set-valued version of Dugundji's extension theorem from Benabdellah and Faik [5], for each $n \geq 1$, there exists some upper semicontinuous extension $\widetilde{F}_{n}$ of $\left.\widetilde{F}\right|_{I_{n} \times H}$ to $I \times H$ that takes on nonempty convex compact values and satisfies, like $\widehat{F}$,

$$
\widetilde{F}_{n}(t, x) \subset \beta(1+\|x\|) K \quad \text { for all }(t, x) \in I \times H .
$$

Since $(\beta+1)^{2}\left(T-T_{0}\right)<m$, due to Theorem 4.1, for each $n \geq 1$, there exist an absolutely continuous map $x_{n}(\cdot): I \rightarrow H$ and an integrable map $z_{n}(\cdot): I \rightarrow H$ such that $x_{n}\left(T_{0}\right)=x_{0}$, and for almost all $t \in I$,

$$
\begin{gathered}
z_{n}(t) \in \widetilde{F}_{n}\left(t, x_{n}(t)\right), \\
-\dot{x}_{n}(t)-z_{n}(t) \in \partial \varphi\left(t, x_{n}(t)\right), \\
\left\|z_{n}(t)\right\| \leq\left(M_{2}+1\right)(\beta+1) \quad \text { and } \quad z_{n}(t) \in\left(M_{2}+1\right)(\beta+1) K,
\end{gathered}
$$

and

$$
\int_{T_{0}}^{T}\left\|\dot{x}_{n}(t)\right\|^{2} d t \leq \alpha_{0}+\sigma\left(T-T_{0}\right)(\beta+1)^{2}\left(1+M_{2}\right)^{2} .
$$

In view of (4.2), we may also write

$$
\int_{T_{0}}^{T}\left\|\dot{x}_{n}(t)\right\|^{2} d t \leq \alpha_{n}+\sigma(\beta+1)^{2} \int_{T_{0}}^{T}\left(1+\left\|x_{n}(t)\right\|\right)^{2} d t,
$$

with

$$
\alpha_{n}=\left(k^{2}(0)+3(\rho+1)^{2}\right) \int_{T_{0}}^{T} \dot{a}^{2}(t) d t+2\left[T-T_{0}+\varphi\left(T_{0}, x_{0}\right)-\varphi\left(T, x_{n}(T)\right)\right] .
$$

(B) Uniform convergence of a subsequence of $\left(x_{n}(\cdot)\right)$ to some map $(x(\cdot))$. In order to prove this, consider the map

$$
Z_{n}(t):=\int_{T_{0}}^{t} z_{n}(s) d s
$$


As in the proof of Theorem 4.1, thanks to (5.9), via Arzela-Ascoli's theorem, we may suppose that the sequence $\left(Z_{n}(\cdot)\right)$ converges uniformly in $\mathcal{C}_{H}(I)$ to some map $Z(\cdot): I \rightarrow H$. Now, let us set

$$
X_{n}(t):=x_{n}(t)+Z_{n}(t) .
$$

We aim at proving that $\left(X_{n}(\cdot)\right)$ is a Cauchy sequence in $\left(\mathcal{C}_{H}(I),\|\cdot\|_{\infty}\right)$. The maps $X_{n}(\cdot)$ are clearly absolutely continuous and for any fixed $p, q \in \mathbb{N}$, and for almost all $t \in\left[T_{0}, T\right]$, one has

$$
\begin{aligned}
\frac{1}{2} \frac{d}{d t} \| X_{p}(t) & -X_{q}(t) \|^{2}=\left\langle\dot{X}_{p}(t)-\dot{X}_{q}(t), X_{p}(t)-X_{q}(t)\right\rangle \\
= & \left\langle\dot{X}_{p}(t)-\dot{X}_{q}(t), x_{p}(t)-x_{q}(t)\right\rangle+\left\langle\dot{X}_{p}(t)-\dot{X}_{q}(t), Z_{p}(t)-Z_{q}(t)\right\rangle .
\end{aligned}
$$

By definition, one has

$$
\begin{aligned}
& -\dot{X}_{p}(t)=-\dot{x}_{p}(t)-z_{p}(t) \in \partial \varphi\left(t, x_{p}(t)\right), \\
& -\dot{X}_{q}(t)=-\dot{x}_{q}(t)-z_{q}(t) \in \partial \varphi\left(t, x_{q}(t)\right),
\end{aligned}
$$

and the monotonicity property of $\partial \varphi(t, \cdot)$ entails that

$$
\left\langle\dot{X}_{p}(t)-\dot{X}_{q}(t), x_{p}(t)-x_{q}(t)\right\rangle \leq 0 .
$$

Therefore, one has

$$
\frac{1}{2} \frac{d}{d t}\left\|X_{p}(t)-X_{q}(t)\right\|^{2} \leq\left\|\dot{X}_{p}(t)-\dot{X}_{q}(t)\right\|\left\|Z_{p}(t)-Z_{q}(t)\right\| .
$$

Now, we deduce from (5.10) that the sequence $\left(\dot{x}_{n}\right)$ is bounded in $L_{H}^{2}(I)$ and since via (5.9)

$$
\sup _{n \in \mathbb{N}}\left\|\dot{Z}_{n}(\cdot)\right\|_{L_{H}^{2}(I)}^{2} \leq\left(T-T_{0}\right)\left(M_{2}+1\right)^{2}(\beta+1)^{2}<+\infty,
$$

we conclude that $A:=\sup _{n \in \mathbb{N}}\left\|\dot{X}_{n}(\cdot)\right\|_{L_{H}^{2}(I)}<+\infty$. The uniform convergence of the sequence $\left(Z_{n}\right)$ assures us that

$$
\int_{T_{0}}^{T}\left\|Z_{p}(t)-Z_{q}(t)\right\| d t \rightarrow 0
$$

when $p, q \rightarrow \infty$. This, along with the fact that $\left\|X_{p}\left(T_{0}\right)-X_{q}\left(T_{0}\right)\right\|=0$, entails, via Lemma 2.1,

$$
\lim _{p, q \rightarrow \infty}\left\|X_{p}(\cdot)-X_{q}(\cdot)\right\|_{\infty}=0 .
$$

Then, the uniform Cauchy's criterion guarantees that the sequence $\left(X_{n}(\cdot)\right)$ converges uniformly on $I$ to some map $X(\cdot) \in \mathcal{C}_{H}(I)$. So, the sequence $\left(x_{n}\right)=$ $\left(X_{n}-Z_{n}\right)$ converges uniformly on $I$ to some continuous map $x(\cdot) \in \mathcal{C}_{H}(I)$, with $x\left(T_{0}\right)=x_{0}$, that is,

$$
x_{n}(\cdot) \rightarrow x(\cdot) \text { strongly in } L_{H}^{2}(I) .
$$


By (5.10) the sequence $\left(\dot{x}_{n}\right)$ is bounded in $L_{H}^{\infty}(I)$ and hence also in $L_{H}^{2}(I)$. We may then, extract a subsequence converging weakly in $L_{H}^{2}(I)$ to some map $v(\cdot)$. The equality

$$
x_{n}(t)=x_{n}\left(T_{0}\right)+\int_{T_{0}}^{t} \dot{x}_{n}(s) d s \quad \text { for all } t \in I
$$

then yields,

$$
x(t)=x\left(T_{0}\right)+\int_{T_{0}}^{t} v(s) d s \quad \text { for all } t \in I
$$

and hence the map $x(\cdot)$ is absolutely continuous on $I$ with $\dot{x}(\cdot)=v(\cdot)$ for almost all $t \in I$ and

$$
\dot{x}_{n}(\cdot) \rightarrow \dot{x}(\cdot) \quad \text { weakly in } L_{H}^{2}(I) .
$$

Due to $(5.9)$, we may also suppose that, for some map $z(\cdot) \in L_{H}^{2}(I)$, one has

$$
z_{n}(\cdot) \rightarrow z(\cdot) \quad \text { weakly in } L_{H}^{2}(I) .
$$

(C) Now, we proceed to prove that $x(\cdot)$ is a solution of $\left(\mathcal{P}_{2}\right)$.

Taking (5.12), (5.14) and (5.15) into account, as in the proof of Theorem 4.1, we have, via the closure property of the subdifferential operator $\partial \varphi(t, \cdot)$, for almost all $t \in I$ the required inclusion, that is,

$$
\dot{x}(t)+z(t) \in-\partial \varphi(t, x(t)) \quad \text { for a.e. } t \in I .
$$

It remains to prove that $z(t) \in F(t, x(t))$ for almost every $t \in I$. Due to (5.15), by Mazur's lemma, there exists a sequence $\left(\zeta_{n}(\cdot)\right)$ in $L_{H}^{1}(I)$ such that

$$
\zeta_{n}(\cdot) \in \operatorname{co}\left\{z_{k}(\cdot): k \geq n\right\} \quad \text { for all } n \geq 1
$$

which converges strongly in $L_{H}^{1}(I)$ to $z(\cdot)$. Thus, extracting a subsequence, we may suppose that $\zeta_{n}(t) \rightarrow z(t)$ for almost every $t \in I$. This, along with (5.17), implies that, for some negligible subset $N_{1} \subset I$,

$$
z(t) \in \bigcap_{n} \overline{\operatorname{co}}\left\{z_{k}(t): k \geq n\right\} \quad \text { for all } t \in I \backslash N_{1} .
$$

Taking (5.7) into account, we may also suppose that, for all $n \geq 1$ and for all $t \in I \backslash N_{1}$,

$$
z_{n}(t) \in \widetilde{F}_{n}\left(t, x_{n}(t)\right)
$$

Consider the $\lambda$-negligible subset $N:=\left(I \backslash \bigcup_{n} I_{n}\right) \cup N_{0} \cup N_{1}$. We are going to prove that $z(t) \in F(t, x(t))$ for all $t \in I \backslash N$. Fix any $\tau \in I \backslash N$. From (5.18) and (5.19), it follows that, for any $\xi \in H$,

$$
\langle\xi, z(\tau)\rangle \leq \limsup _{n} \sigma\left(\widetilde{F}_{n}\left(\tau, x_{n}(\tau)\right), \xi\right) .
$$


On the other hand, by definition of $N$, there exists an integer $n(\tau)$ such that $\tau \in I_{n(\tau)} \backslash N_{0}$ and, $\left(I_{n}\right)$ being increasing, one has $\tau \in I_{n}$ for all $n \geq n(\tau)$. Consequently, for all $n \geq n(\tau)$,

$$
\widetilde{F}_{n}\left(\tau, x_{n}(\tau)\right)=\widetilde{F}\left(\tau, x_{n}(\tau)\right) \subset \widehat{F}\left(\tau, x_{n}(\tau)\right),
$$

the inclusion coming from (5.6). Note that, by (5.10), and taking (5.4) into account, one has, for all $n \geq 1$ and for almost all $t \in I,\left\|x_{n}(t)\right\| \leq M_{2}$, and hence, thanks to (5.5), for all $n \geq 1$,

$$
\widehat{F}\left(\tau, x_{n}(\tau)\right)=F\left(\tau, x_{n}(\tau)\right) .
$$

Therefore, due to $(5.20)-(5.22)$ and the fact that $F(\tau, \cdot)$ is scalarly upper semicontinuous, we have

$$
\langle\xi, z(\tau)\rangle \leq \sigma(F(\tau, x(\tau)), \xi) .
$$

This being true for any $\xi \in H$, and $F(\tau, x(\tau))$ being closed and convex, it results that $z(\tau) \in F(\tau, x(\tau))$. Since the latter is satisfied for any $\tau \in I \backslash N$, one has

$$
z(t) \in F(\tau, x(t)) \quad \text { for a.e. } t \in I .
$$

This, along with (5.16) and the fact that $x\left(T_{0}\right)=\lim _{n} x_{n}\left(T_{0}\right)=x_{0}$, proves that $x(\cdot)$ is a solution of $\left(\mathcal{P}_{2}\right)$. Finally, taking the superior limit on $n$ in $(5.11)$, as in the proof of Theorem 4.1, we get the required inequality.

Actually, we have the following more general result. Here, the growth condition involves an $L_{\mathbb{R}}^{1}(I)$ function instead of a constant.

THEOREM 5.2. Under assumptions of Theorem 4.1 on $H$ and $\varphi$, let $F: I \times$ $H \rightrightarrows H$ be a set-valued mapping with nonempty convex compact values such that

(a) for any $x \in H, F(\cdot, x)$ has a $\lambda$-measurable selection;

(b) for all $t \in I, F(t, \cdot)$ is scalarly upper semicontinuous on $H$;

(c) for some compact subset $K \subset \mathbb{B}$ and for some non-negative function $\beta(\cdot) \in L_{\mathbb{R}}^{1}(I)$, for all $(t, x) \in I \times H$, one has

$$
F(t, x) \subset \beta(t)(1+\|x\|) K .
$$

Then, for any $x_{0} \in \operatorname{dom} \varphi\left(T_{0}, \cdot\right)$ the following problem

$$
\left\{\begin{array}{l}
-\dot{x}(t) \in \partial \varphi(t, x(t))+F(t, x(t)) \quad \text { for a.e. } t \in I, \\
x\left(T_{0}\right)=x_{0}
\end{array}\right.
$$

has at least one absolutely continuous solution. More precisely, there exist an absolutely continuous map $x(\cdot): I \rightarrow H$ and an integrable map $z(\cdot): I \rightarrow H$ such that $x\left(T_{0}\right)=x_{0}, x(t) \in \operatorname{dom} \varphi(t, x(t))$ for all $t \in I$, and for almost all $t \in I, z(t) \in F(t, x(t))$ and $-\dot{x}(t)-z(t) \in \partial \varphi(t, x(t))$ and

$$
z(t) \in 2(\beta(t)+1)(\|x(t)\|+1) \overline{\operatorname{co}}(K \cup\{0\}) .
$$


Proof. We suppose without loss of generality, that $K$ is convex and contains 0 . If not so, we may replace $K$ by $\overline{c o}(K \cup\{0\})$. Suppose further,

$$
\int_{T_{0}}^{T}(\beta(s)+1) d s<\frac{1}{2}\left(T-T_{0}\right)^{1 / 2} m^{1 / 2} .
$$

(A) Following an idea from Deimling [16], let us set $\widehat{T}:=\int_{T_{0}}^{T}(\beta(s)+1) d s$ and let us define an absolutely continuous function $\widehat{\beta}(\cdot):\left[T_{0}, T\right] \rightarrow[0, \widehat{T}]$ by

$$
\widehat{\beta}(t):=\int_{T_{0}}^{t}(\beta(s)+1) d s .
$$

Thanks to the fact that $\beta(t)+1>0$ for almost all $t \in I$, the absolutely continuous function $\widehat{\beta}(\cdot)$ is increasing and hence has a continuous inverse function $\widehat{\beta}^{-1}(\cdot):[0, \widehat{T}] \rightarrow\left[T_{0}, T\right]$. Notice that $\widehat{\beta}^{-1}(\cdot)$ is Lipschitz on $[0, \widehat{T}]$. Indeed, for $\widehat{t}, \widehat{s} \in[0, \widehat{T}]$ with $\widehat{s} \leq \widehat{t}$ there exist $t, s \in\left[T_{0}, T\right]$ with $s \leq t$ such that $\widehat{t}=\widehat{\beta}(t)$ and $\widehat{s}=\widehat{\beta}(s)$, and then, using (5.24), one has

$$
\widehat{\beta}^{-1}(\widehat{t})-\widehat{\beta}^{-1}(\widehat{s})=t-s \leq \int_{s}^{t}(\beta(\tau)+1) d \tau=\widehat{\beta}(t)-\widehat{\beta}(s)=\widehat{t}-\widehat{s} .
$$

This yields that, for any $\widehat{t}, \widehat{s} \in[0, \widehat{T}], \widehat{\beta}^{-1}(\widehat{t})-\widehat{\beta}^{-1}(\widehat{s}) \leq \widehat{t}-\widehat{s}$, which means that $\widehat{\beta}^{-1}(\cdot)$ is Lipschitz on $[0, \widehat{T}]$.

Now, consider the set-valued map $\widehat{F}:[0, \widehat{T}] \times H \rightrightarrows H$ defined by

$$
\widehat{F}(t, x):=\frac{1}{\beta\left(\widehat{\beta}^{-1}(t)\right)+1} F\left(\widehat{\beta}^{-1}(t), x\right) .
$$

Clearly, like $F$, the set-valued map $\widehat{F}$ satisfies the conditions (a) and (b) of Theorem 5.1 and, by (c), for all $(t, x) \in[0, \widehat{T}] \times H$,

$$
\widehat{F}(t, x) \subset(1+\|x\|) K .
$$

Consider also the single valued map $\widehat{\varphi}:[0, \widehat{T}] \times H \rightarrow[0,+\infty]$ defined by

$$
\widehat{\varphi}(t, x):=\varphi\left(\widehat{\beta}^{-1}(t), x\right) .
$$

Obviously, $\widehat{\varphi}$ satisfies assumptions $\left(\mathrm{H}_{1}\right)$ and $\left(\mathrm{H}_{2}\right)$. Therefore, from the previous result, there exist an absolutely continuous map $X(\cdot):[0, \widehat{T}] \rightarrow H$ and an integrable map $\widehat{z}(\cdot):[0, \widehat{T}] \rightarrow H$ such that $X(0)=x_{0}$ and, for almost all $t \in[0, \widehat{T}]$,

$$
\left\{\begin{array}{l}
\widehat{z}(t) \in \widehat{F}(t, X(t)) \\
-\dot{X}(t) \in \partial \widehat{\varphi}(t, X(t))+\widehat{z}(t) .
\end{array}\right.
$$

By inequality (5.3), along with (5.26), one has

$$
\int_{0}^{\widehat{T}}\|\dot{X}(t)\|^{2} d t \leq \alpha+4 \sigma \int_{0}^{\widehat{T}}(1+\|X(t)\|)^{2} d t .
$$


Then

$$
\begin{aligned}
\int_{0}^{\widehat{T}}\|\dot{X}(t)\|^{2} d t & \leq \alpha+4 \sigma\left(1+\|X(\cdot)\|_{\infty}\right)^{2} \int_{0}^{\widehat{T}} d t \\
& \leq \alpha+4 \sigma\left(1+\|X(\cdot)\|_{\infty}\right)^{2} \widehat{T} \leq \alpha+8 \sigma\left(1+\|X(\cdot)\|_{\infty}^{2}\right) \widehat{T},
\end{aligned}
$$

where $\|\cdot\|_{\infty}$ denotes the supremum norm over the interval $[0, \widehat{T}]$.

Using the Cauchy-Schwarz inequality, one has, for all $s \in[0, \widehat{T}]$,

$$
\begin{aligned}
\|X(s)-X(0)\|^{2} & \leq s\left(\int_{0}^{s}\|\dot{X}(t)\|^{2} d t\right) \leq \widehat{T}\left(\alpha+8 \sigma\left(1+\|X(\cdot)\|_{\infty}^{2}\right) \widehat{T}\right) \\
\|X(s)\|^{2} & \leq 2\left\|x_{0}\right\|^{2}+2\left\|X(s)-x_{0}\right\|^{2} \\
& \leq 2\left\|x_{0}\right\|^{2}+2 \widehat{T}\left(\alpha+8 \sigma\left(1+\|X(\cdot)\|_{\infty}^{2}\right) \widehat{T}\right) .
\end{aligned}
$$

Then $\left(1-16 \sigma \widehat{T}^{2}\right)\|X(\cdot)\|_{\infty}^{2} \leq 2\left(\left\|x_{0}\right\|^{2}+\widehat{T}(\alpha+8 \sigma \widehat{T})\right)$. Therefore, taking (5.23) into account, that is, $16 \sigma \widehat{T}^{2}<1$, one has $\|X(\cdot)\|_{\infty} \leq M_{3}$, where

$$
M_{3}:=\left(\frac{2\left(\left\|x_{0}\right\|^{2}+\widehat{T}(\alpha+8 \sigma \widehat{T})\right)}{1-16 \sigma \widehat{T}^{2}}\right)^{1 / 2} .
$$

Consequently, inclusion (5.1) of Theorem 5.1 yields $(\beta=1), \widehat{z}(t) \in 2\left(1+M_{3}\right) K$.

(B) Let us prove that the absolutely continuous map $x(\cdot):\left[T_{0}, T\right] \rightarrow H$ defined, for any $t \in\left[T_{0}, T\right]$, by $x(t)=X(\widehat{\beta}(t))$ is a solution of $\left(\mathcal{P}_{3}\right)$.

Let us set $I_{1}:=\left\{t \in\left[T_{0}, T\right]: \dot{\widehat{\beta}}(t)\right.$ exists $\}$ and $I_{2}:=\{\widehat{t} \in[0, \widehat{T}]: \dot{X}(\widehat{t})$ exists and (5.27) holds at $\widehat{t}\}$. Consider the subsets $N_{1}:=\left[T_{0}, T\right] \backslash I_{1}$ and $\widehat{N}_{2}:=[0, \widehat{T}] \backslash I_{2}$, which are $\lambda$-negligible, and put

$$
N_{2}:=\left\{t \in\left[T_{0}, T\right]: \widehat{\beta}(t) \in \widehat{N}_{2}\right\}=\widehat{\beta}^{-1}\left(\widehat{N}_{2}\right) .
$$

As $\widehat{\beta}^{-1}(\cdot)$ is Lipschitz on $[0, \widehat{T}]$, the set $N_{2}$ is also $\lambda$-negligible. So, $N:=N_{1} \cup N_{2}$ is $\lambda$-negligible and, for any $t \in\left[T_{0}, T\right] \backslash N$,

$$
\dot{x}(t)=\dot{\widehat{\beta}}(t) \dot{X}(\widehat{\beta}(t))=(\beta(t)+1) \dot{X}(\widehat{\beta}(t)) .
$$

The definitions of the negligible sets above, along with (5.25) and (5.27), entail that, for all $t \in\left[T_{0}, T\right] \backslash N$,

$$
\left\{\begin{array}{l}
\widehat{z}(\widehat{\beta}(t)) \in \frac{1}{\beta(t)+1} F(t, x(t)), \\
-\dot{X}(\widehat{\beta}(t)) \in \partial \varphi(t, x(t))+\widehat{z}(\widehat{\beta}(t)) .
\end{array}\right.
$$

Hence, defining $z(\cdot):\left[T_{0}, T\right] \rightarrow H$ by $z(t):=(\beta(t)+1) \widehat{z}(\widehat{\beta}(t))$, we obtain, by (5.29), for any $t \in\left[T_{0}, T\right] \backslash N$, and

$$
\left\{\begin{array}{l}
z(t) \in F(t, x(t)), \\
-\dot{x}(t) \in \partial \varphi(t, x(t))+z(t),
\end{array}\right.
$$

which ends the proof. 
REMARK 5.3. Under conditions of Theorem 5.1 or Theorem 5.2, estimates and inclusions in Proposition 4.2 hold true.

Acknowledgments. The authors would like to thank Professor Lionel Thibault for his valuable comments and suggestions.

\section{REFERENCES}

[1] H. Aтtouch, Variational convergence for functions and operators, Applicable Mathematics Series, Pitman, Boston, London, Melbourne, 1984.

[2] H. Attouch and A. Damlamian, On Multivalued evolution equations in Hilbert apaces, Israel J. Math. 12 (1972), 373-390.

[3] H. Attouch and A. Damlamian, Strong solutions for parabolic variational inequalities, Nonlinear Anal. 2 (1978), 329-353.

[4] J.P. Aubin And A. Cellina, Differential Inclusions, Set-Valued Maps and Viability Theory, Springer-Verlag, Berlin, Heidelberg, 1984.

[5] H. Benabdellah and A. Faik, Perturbations convexes et non convexes des équations d'évolution Portugal. Math. 53 (1996), Fasc. 2, 187-208.

[6] H. Benabdellah, C. Castaing and A. Salvadori, Compactness and discretization methods for differential inclusions and evolution problems, Atti Sem. Math. Fis. Univ. Modena XLV (1997), 9-51.

[7] M. Bounkhel and L. Thibault, Nonconvex sweeping process and prox-regularity in Hilbert space, J. Nonlinear Convex Anal. 6 (2005), no. 2, 359-374.

[8] C. Castaing, L. Aicha Faik and A. Salvadori, Evolution equations governed by maccretive and subdifferential operators with delay, Int. J. Appl. Math. 2 (2000), no. 9, 1005-1026.

[9] C. Castaing, A.G. Ibrahim and M.F. Yarou, Existence problems in second order evolution inclusions: Discretization and variational approch, Taiwanese J. Math. 12 (2008), no. $6,1435-1477$.

[10] _ Some contributions to nonconvex sweeping process, J. Nonlinear Convex Anal. 10, (2009), no. 1, 1-20.

[11] C. Castaing and S. Marcellin, Evolution inclusions with pln functions and application to viscosity and control, J. Nonlinear Convex Anal. 8 (2007), no. 2, 227-255.

[12] C. Castaing and M.D.P. Monteiro Marques, Evolution problems associated with nonconvex closed moving sets with bounded variation, Portugal. Math. 53 (1996), Fasc. 2, $73-78$.

[13] C. Castaing, A. Salvadori and L. Thibault, Functional evolution equations governed by nonconvex sweeping process, J. Nonlinear Convex Anal. 2 (2001), no. 2, 217-241.

[14] C. Castaing and M. Valadier, Convex Analysis and Measurable Multifunctions, Lecture Notes in Math 580, Springer-Verlag, Berlin, Heidelberg (1977).

[15] A. Cellina And V. Staicu, On evolution equations having monotonicities of opposite sign, J. Differential Equations 90 (1991), 71-80.

[16] K. Deimling, Multivalued Differential Equations, De Gruyter, New York, 1992.

[17] N. Dunford and J.T. Schwartz, Linear Operators, Part I, Interscience Publishers, Inc., New York, 1967.

[18] J.F. Edmond, Problèmes d'évolution associés à des ensembles prox-réguliers. Inclusions et intégration de sous-différentiels, Thesis, Université Montpellier II, 2004.

[19] J.F. Edmond And L. Thibault, Relaxation of an optimal control problem involving a perturbed sweeping process, Math. Program Ser. B 104 (2005), 347-373. 
$[20] \_, B V$ solution of nonconvex sweeping process differential inclusion with perturbation, J. Differential Equations 226 (1) (2006), 135-159.

[21] N. Kenmochi, Some nonlinear parabolic variational inequalities, Israel J. Math. 22 (1975), 304-331.

[22] M. KuBo, Caracterisation of a class of evolution operators generated by time dependent subdifferential, Funkcial. Ekvac. 32 (1989), 301-321.

[23] M. OtAni, Nonmonotone perturbations for nonlinear parabolic equations associated with subdifferential operators, Cauchy problems, J. Differential Equations 46, (1982), 268-299.

[24] J.C. PeralBA, Équations d'évolution dans un espace de Hilbert, associées à des opérateurs sous-différentiels, Thèse de doctorat de spécialité, Montpellier, 1973.

[25] _ Un problème d'évolution relatif à un opérateur sous différentiel dépendant du temps, Séminaire d'analyse convexe, Montpellier, exposé No. 6, (1972).

[26] S. Saïdi, L. Thibault And M.F. Yarou, Relaxation of optimal control problems involving time dependent subdifferential operators, Numer. Funct. Anal. Optim. 34 (10) (2013), $1156-1186$.

[27] A.A. Tolstonogov, Properties of attainable sets of evolution inclusions and control systems of subdifferential type, Sib. Math. J. 45 (2004), no. 4, 763-784.

[28] J. Watanabe, On certain nonlinear evolution equations, J. Math. Soc. Japan 25, (1973), 446-463.

[29] Y. Yamada, On evolution equations generated by subdifferential operators, J. Math. Sci. Univ. Tokyo 23 (1976), 491-515.

[30] N. Yamazaki, Attractors of asymptotically periodic multivalued dynamical systems governed by time-dependent subdifferentials, Electron. J. Differential Equations 107, (2004), $1-22$.

[31] S. Yotsutani, Evolution equations associated with subdifferentials, J. Math. Soc. Japan 31 (1978), 623-646.

Soumia Saïdi and Mustapha Fateh Yarou

Laboratoire LMPA

Department of Mathematics

Jijel University, ALGERIA

E-mail address: soumia_ss@hotmail.fr,mfyarou@yahoo.com 